\title{
Altered Autophagy-Associated Genes Expression in T Cells of Oral Lichen Planus Correlated with Clinical Features
}

\author{
Ya-Qin Tan, ${ }^{1}$ Jing Zhang, ${ }^{1,2}$ Ge-Fei Du, ${ }^{1,2}$ Rui Lu, ${ }^{1,2}$ Guan-Ying Chen, ${ }^{1}$ and Gang Zhou ${ }^{1,2}$ \\ ${ }^{1}$ The State Key Laboratory Breeding Base of Basic Science of Stomatology (Hubei-MOST) and Key Laboratory of \\ Oral Biomedicine Ministry of Education, School and Hospital of Stomatology, Wuhan University, Luoyu Road 237, \\ Wuhan 430079, China \\ ${ }^{2}$ Department of Oral Medicine, School and Hospital of Stomatology, Wuhan University, Luoyu Road 237, Wuhan 430079, China
}

Correspondence should be addressed to Gang Zhou; gordonzhou@tom.com

Received 10 November 2015; Revised 13 January 2016; Accepted 13 January 2016

Academic Editor: Mirella Giovarelli

Copyright (C) 2016 Ya-Qin Tan et al. This is an open access article distributed under the Creative Commons Attribution License, which permits unrestricted use, distribution, and reproduction in any medium, provided the original work is properly cited.

\begin{abstract}
Oral lichen planus (OLP) is a T cell-mediated inflammatory autoimmune disease. Autophagy has emerged as a fundamental trafficking event in mediating $\mathrm{T}$ cell response, which plays crucial roles in innate and adaptive immunity. The present study mainly investigated the mRNA expression of autophagy-associated genes in peripheral blood T cells of OLP patients and evaluated correlations between their expression and the clinical features of OLP. Five differentially expressed autophagy-associated genes were identified by autophagy array. Quantitative real-time RT-PCR results confirmed that IGF1 expression in the peripheral blood T cells of OLP patients was significantly higher than that in controls, especially in female and middle-aged (30-50 years old) OLP patients. In addition, $A T G 9 B$ mRNA levels were significantly lower in nonerosive OLP patients. However, no significant differences were found in the expression of HGS, ESR1, and SNCA between OLP patients and controls. Taken together, dysregulation of T cell autophagy may be involved in immune response of OLP and may be correlated with clinical patterns.
\end{abstract}

\section{Introduction}

Oral lichen planus (OLP) is a common $\mathrm{T}$ cell-mediated chronic inflammatory disease with the characteristics of adult onset, female predilection, and autoimmune attack by infiltrating $\mathrm{T}$ cells in the oral mucosa and extraoral lesions on areas including the skin, genitalia, and nails $[1,2]$. OLP affects $1-2 \%$ of the general adult population and is considered as a potentially malignant disorder with a malignant transformation rate of $0-1 \%$ according to the World Health Organization (WHO) [3]. Clinically, OLP mainly manifests three different forms: reticular, atrophic, and erosive [4]. They are generally simplified into two categories: erosive (erosive lesions) and nonerosive (reticular and atrophic lesions) [5]. Histologically, OLP is characterized by a dense infiltration of $\mathrm{T}$ cells in the lamina propria, basement membrane disruption, and the degeneration of basal keratinocytes [4, 6]. Extensive evidence has suggested that the $\mathrm{T}$ cell-mediated immune responses have pivotal roles in the onset and perpetuation of this disorder [4, 6-9]. The immunopathogenesis of OLP may involve antigen presentation, $\mathrm{T}$ cell activation and migration, and keratinocyte apoptosis [4]. In OLP, T cells are activated when presented with antigens by major histocompatibility complex (MHC) classes II and I molecules [3, 4]. Following antigen recognition, activated cytotoxic $\mathrm{T}$ cells may trigger keratinocyte apoptosis and release chemokines that attract additional helper $\mathrm{T}$ cells into the developing OLP lesion. The activated helper $\mathrm{T}$ cells may in turn activate cytotoxic $\mathrm{T}$ cells and participate in the antigen presentation and keratinocyte apoptosis $[2,3]$. Our previous studies revealed that an imbalance between Th1/Th2 immune response was associated with disease onset and showed that OLP was featured by a Thl cytokine bias and a Thl-biased pattern of upstream transcription factors expression. Thus, the Th1 immune response plays a dominant role in $\operatorname{OLP}[4,6,7]$.

As a strictly regulated lysosomal degradation pathway, autophagy is crucial for maintaining intracellular homeostasis and normal development [10]. Autophagy is involved in various innate and adaptive immune processes, including pathogen recognition and destruction, antigen processing for 
MHC presentation, lymphocyte development and function, and inflammatory regulation [11, 12]. After being stimulated by $\mathrm{T}$ cell receptor activation upon antigen recognition, autophagy can be induced in $\mathrm{T}$ cells and is required for $\mathrm{T}$ cell proliferation, differentiation, survival, and death $[13,14]$. Autophagy has emerged as a fundamental trafficking event in mediating $\mathrm{T}$ cell response and regulating $\mathrm{T}$ cell immunity $[13,15]$. Thus, $\mathrm{T}$ cell autophagy is hypothesized to be involved in the immunopathogenesis of OLP.

Defects in autophagy-associated genes and recruitment of autophagy-associated proteins are essential for autophagic dysfunction [16]. In recent years, the dysregulation of autophagy-associated genes has been recognized to increase the susceptibility to diverse diseases, including inflammation, autoimmune disorders, and cancer [17-19]. Barrett et al. reported that three autophagy-associated genes (IRGM, NOD2, and ATG16L1) were involved in autoinflammatory Crohn's disease [20]. Besides, elevated expression of ATG5 in $\mathrm{T}$ cells may contribute to the $\mathrm{T}$ cell-mediated inflammatory demyelination in multiple sclerosis [21]. Furthermore, aberrant ATG5, ATG7, LC3, HSPA/HSP70, UVRAG, and IRGM expression levels have been implicated in the dysregulated $\mathrm{T}$ cell immune response of systemic lupus erythematosus [22]. These findings have suggested the immunological role of $\mathrm{T}$ cell autophagy in human diseases. However, it is not yet known whether autophagy in $\mathrm{T}$ cells plays a role in the pathogenesis of OLP. Therefore, reverse transcription polymerase chain reaction (RT-PCR) autophagy arrays were performed using RNA extracted from the peripheral blood $\mathrm{T}$ cells of OLP patients to screen 84 genes that encode components of the molecular machinery and key regulators of autophagy. Furthermore, differential gene expression between OLP patients and controls was validated using quantitative real-time RT-PCR methods. Finally, correlations between the mRNA expression of these autophagy-associated genes and the clinical features of OLP were analyzed.

\section{Materials and Methods}

2.1. Study Participants. Twenty-five patients with OLP and 13 age-sex-matched healthy controls were recruited from the Department of Oral Medicine, School and Hospital of Stomatology, Wuhan University. Informed consent was obtained from each subject before the study began. The Ethical Committee Board of the School and Hospital of Stomatology, Wuhan University, approved this study according to the Declaration of Helsinki on human subject protection. The group of OLP patients consisted of 13 females and 12 males with a mean age of 40.2 years and an age range of 19-57 $(40.2 \pm 2.4$ years). There were 8 females and 5 males with an age range of $20-55$ ( $34.5 \pm 3.3$ years $)$ in the control group. The subjects were enrolled according to the criteria described in our previous studies [4]. Briefly, all study subjects did not suffer any other disorders or receive any treatment within recent 3 months. In addition, OLP can be subdivided into nonerosive form (NEOLP) and erosive form (EOLP). The clinical characteristics of the study subjects were listed in Table 1 .
TABLE 1: Clinical features of the subjects.

\begin{tabular}{lcc}
\hline & OLP $(n=25)$ & Control $(n=13)$ \\
\hline Gender & & \\
Male & 12 & 5 \\
Female & 13 & 8 \\
Age (years) & & $20 \sim 55$ \\
$\quad$ Range & $19 \sim 57$ & $34.5 \pm 3.3$ \\
Mean \pm SD & $40.2 \pm 2.4$ & - \\
Clinical form & & \\
Nonerosive & 12 & \\
Erosive & 13 & \\
\hline
\end{tabular}

2.2. T Cells Isolation and Purification. Peripheral blood mononuclear cells (PBMCs) were collected from subjects by venipuncture and separated by density-gradient centrifugation over Ficoll-Hypaque solution (Tianjin Haoyang Biological Manufacture Co. Ltd., Tianjin, China). T cells were negatively selected from PBMCs using biotin human T lymphocyte enrichment cocktail and streptavidin particles (BD Biosciences, San Jose, CA, USA) together with an IMag ${ }^{\text {ma }}$ cell separation system (BD Biosciences).

2.3. RNA Extraction. Total RNA was extracted from $1 \times$ $10^{7} \mathrm{~T}$ cells using an $\mathrm{RNeasy}^{\circledast}$ mini kit (QIAGEN GmbH, Hilden, Germany). Potential genomic DNA contamination was removed from the samples by treatment with RNase-free DNase (QIAGEN) for $15 \mathrm{~min}$ at room temperature. Concentration and purity were determined using a NanoDrop $1000^{\mathrm{TM}}$ spectrophotometer (Thermo Fisher, Dubuque, IA, USA). The quality of the isolated RNA samples was confirmed by examining the integrity of $28 \mathrm{~S}$ and $18 \mathrm{~S}$ ribosomal RNA bands through electrophoresis on agarose gels containing formaldehyde.

2.4. Autophagy Array Assay. The human $\mathrm{RT}^{2}$ profiler PCR autophagy array (QIAGEN) was used to study the expression of 84 autophagy-associated genes in the peripheral blood $\mathrm{T}$ cells of OLP patients and controls. Briefly, using an $\mathrm{RT}^{2}$ first Strand kit (QIAGEN), $1 \mu$ g total RNA obtained from T cells was incubated with the kit's genomic DNA elimination mixture at $42^{\circ} \mathrm{C}$ for $5 \mathrm{~min}$ and then transferred to ice for no less than $1 \mathrm{~min}$ to remove any residual DNA contamination. The kit's reverse transcription mixture was added to the purified RNA sample. The mixture was incubated at $42^{\circ} \mathrm{C}$ for $15 \mathrm{~min}$ and then $95^{\circ} \mathrm{C}$ for $5 \mathrm{~min}$ to convert total RNA back into cDNA. After cDNA synthesis, real-time RTPCR was performed using $\mathrm{RT}^{2} \mathrm{SYBR}^{\circledR}$ Green master mix (QIAGEN), according to the manufacturer's instructions. The amplification data (fold changes in the threshold cycle [Ct] values of all the genes) were analyzed by the $\Delta \Delta \mathrm{Ct}$ method.

2.5. Quantitative Real-Time RT-PCR Confirmation. Next, we inspected 5 autophagy-associated genes that were identified to be differentially expressed between OLP patients and controls by the autophagy array: insulin-like growth factor 1 
TABLE 2: Primer pairs designed for quantitative real-time RT-PCR analysis.

\begin{tabular}{|c|c|c|c|}
\hline Gene & GenBank accession number & Primer sequences & PCR product size (bp) \\
\hline \multirow{2}{*}{$I G F 1$} & \multirow{2}{*}{ NM_000618 } & Forward: 5'-TCCTCGCATCTCTTCTACCTG-3' & \multirow{2}{*}{153} \\
\hline & & Reverse: 5'-ATACCCTGTGGGCTTGTTGA-3' & \\
\hline \multirow{2}{*}{ ATG9B } & \multirow{2}{*}{ NM_173681 } & Forward: $5^{\prime}$-CCTCGTGCCCTGGAGATTA-3' & \multirow{2}{*}{196} \\
\hline & & Reverse: 5' -AGAACCGCATCAAAGAAAGC-3' & \\
\hline \multirow{2}{*}{$H G S$} & \multirow{2}{*}{ NM_004712 } & Forward: $5^{\prime}$-AGTCTGAGGAGAGCCACGAG-3' & \multirow{2}{*}{114} \\
\hline & & Reverse: 5'-CCGAGTCATTGGTGATGCT-3' & \\
\hline \multirow{2}{*}{ ESR1 } & \multirow{2}{*}{ NM_000125 } & Forward: 5'-GGCTACATCATCTCGGTTCC-3' & \multirow{2}{*}{118} \\
\hline & & Reverse: 5'-AGACTTCAGGGTGCTGGACA-3' & \\
\hline \multirow{2}{*}{ SNCA } & \multirow{2}{*}{ NM_000345 } & Forward: $5^{\prime}$-GGGCAAGAATGAAGAAGGAG-3' & \multirow{2}{*}{150} \\
\hline & & Reverse: 5'-CAAGAAACTGGGAGCAAAGA-3' & \\
\hline \multirow{2}{*}{ GAPDH } & \multirow{2}{*}{ NM_001289746 } & Forward: $5^{\prime}$-CTTTGGTATCGTGGAAGGACTC-3' & \multirow{2}{*}{134} \\
\hline & & Reverse: $5^{\prime}$-CAGTAGAGGCAGGGATGATGTT-3' & \\
\hline
\end{tabular}

(IGF1), autophagy related 9 homolog B (ATG9B), hepatocyte growth factor-regulated tyrosine kinase substrate (HGS), estrogen receptor 1 (ESR1), and synuclein alpha (SNCA). cDNA was synthesized from total RNA using a PrimeScript ${ }^{\text {TM }}$ RT reagent kit with gDNA Eraser (Takara Bio, Dalian, China). The analysis of these genes expression was done with SYBR Premix Ex Taq ${ }^{\mathrm{Tm}}$ II (Takara) using CFX96 ${ }^{\mathrm{TM}}$ realtime PCR detection system (Bio-Rad Laboratories, Hercules, CA, USA) in a 96-well optical plate. The PCR conditions were $95^{\circ} \mathrm{C}$ for $30 \mathrm{~s}$, followed by 40 cycles of $5 \mathrm{~s}$ at $95^{\circ} \mathrm{C}$ and $30 \mathrm{~s}$ at $60^{\circ} \mathrm{C}$. The expression value for each gene was normalized to the expression level of human glyceraldehyde3-phosphate dehydrogenase $(G A P D H)$, which was used as an internal control. The forward primer and reverse primer were designed (Sangon ${ }^{\mathrm{TM}}$ Biotech, Shanghai, China) as shown in Table 2. The Ct values of 3 replicates for all examined genes and the internal control per sample were used to calculate $2^{-\Delta \Delta \mathrm{Ct}}$ values.

2.6. Statistical Analysis. All calculations were performed by independent-samples $t$-test and one-way ANOVA analysis of variance using SPSS statistical software (SPSS 17.0; SPSS Inc., Chicago, IL, USA). Data were presented as means \pm SEM, and statistical significance was defined as $p<0.05$.

\section{Results}

3.1. Bioinformatics Analysis of RT-PCR Autophagy Array Results. The relative expression levels of the genes which met the criteria fold regulation $>2$ or $<-2$ and fold change $>2$ or $<0.5$ were considered to be significantly different between OLP patients and controls. As shown in Figure 1, the expression of 84 autophagy-associated genes was measured in peripheral blood $\mathrm{T}$ cells of all subjects. Application of the criteria yielded a total of 5 differentially expressed genes. An increased expression of $I G F 1$ (fold regulation $=2.74$; fold change $=2.74$ ) and a decreased expression of $A T G 9 B$ (fold regulation $=-2.25$; fold change $=0.44$ ), HGS (fold regulation $=-2.43$; fold change $=0.41$ ), ESR1 (fold regulation $=-2.04$; fold change $=0.49$ ), and $S N C A$ (fold regulation $=-2.31$; fold change $=0.43$ ) was found in the peripheral blood $\mathrm{T}$ cells of OLP patients. The results of hierarchical clustering analysis also indicated that these autophagy-associated genes were differentially expressed among patients with erosive and nonerosive OLP and controls (Figure 2).

3.2. Confirmation of the Differential Expression of IGF1, ATG9B, HGS, ESR1, and SNCA in T Cells between OLP Patients and Controls. Next, confirmation of the autophagy array results was performed by quantitative real-time RTPCR. The expression of IGF1 mRNA was increased in the peripheral blood $\mathrm{T}$ cells of OLP patients, compared with that in the controls (OLP versus control: $1.78 \pm 0.33$ versus $0.52 \pm 0.1, p=0.001)$. However, there were no significant differences in the expression of ATG9B, HGS, ESR1, and $S N C A$ between the OLP group $(n=22)$ and control group $(n=10)$ (Figure 3(a)).

3.3. Expression of IGF1, ATG9B, HGS, ESR1, and SNCA in $T$ Cells from Patients with Different Clinical Forms of OLP. The results showed that $A T G 9 B$ was differentially expressed between erosive and nonerosive OLP patients (Figure 3(b)). Overall, ATG9B mRNA expression was decreased in nonerosive OLP patients compared with that in controls and erosive OLP patients (nonerosive OLP versus control: $0.56 \pm 0.09$ versus $1.71 \pm 0.49, p=0.044$; nonerosive OLP versus erosive OLP: $0.56 \pm 0.09$ versus $1.34 \pm 0.18, p=0.001$ ). There were no significant differences in the expression levels of IGF1, HGS, ESR1, and SNCA between erosive $(n=11)$ and nonerosive $\operatorname{OLP}(n=11)$ patients (Figures $3(\mathrm{c})-3(\mathrm{f}))$.

3.4. Differences in the Expression of IGF1, ATG9B, HGS, ESR1, and SNCA in T Cells between Male and Female OLP Patients. Interestingly, IGF1 mRNA expression in female OLP patients was obviously increased than that in female controls (OLP versus control: $2.43 \pm 0.45$ versus $0.64 \pm 0.18, p=0.042$ ) (Figure 4(a)). IGF1 mRNA expression in female OLP patients was also higher than that in male OLP patients (Female OLP versus Male OLP: $2.43 \pm 0.45$ versus $0.98 \pm 0.36$, $p=0.024$ ) (Figure 4(b)). However, ATG9B, HGS, ESR1, and 
$\begin{array}{llllllllllll}01 & 02 & 03 & 04 & 05 & 06 & 07 & 08 & 09 & 10 & 11 & 12\end{array}$

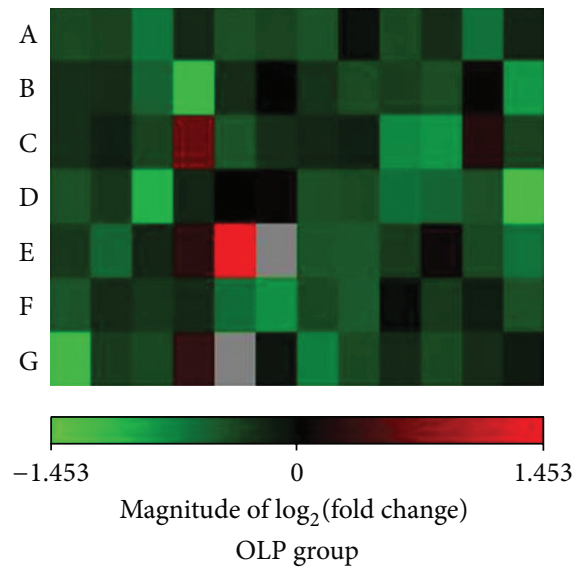

$\begin{array}{llllllllllll}01 & 02 & 03 & 04 & 05 & 06 & 07 & 08 & 09 & 10 & 11 & 12\end{array}$

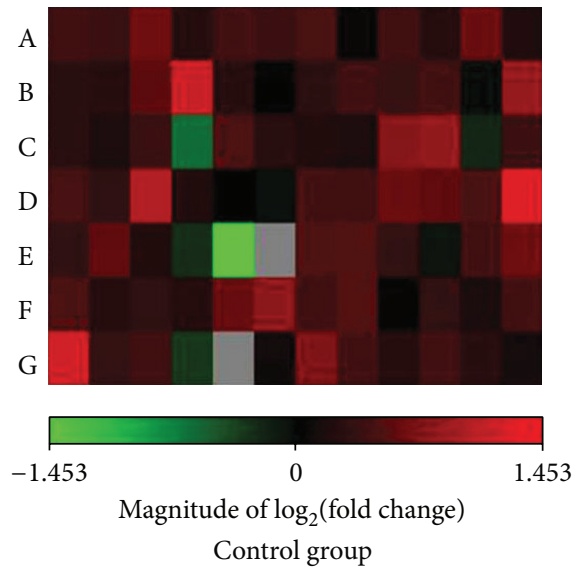

(a)

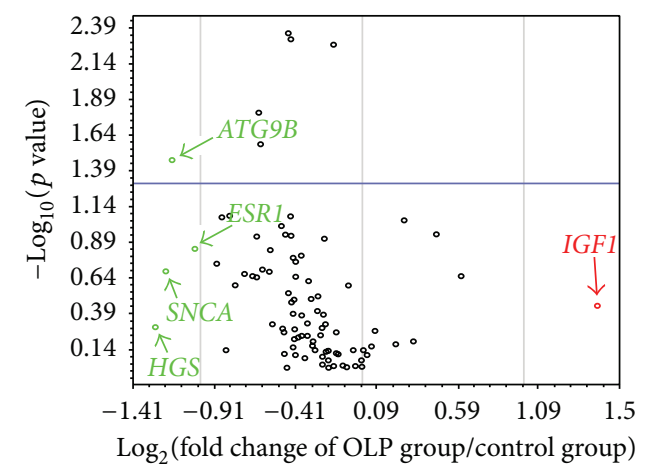

(c)

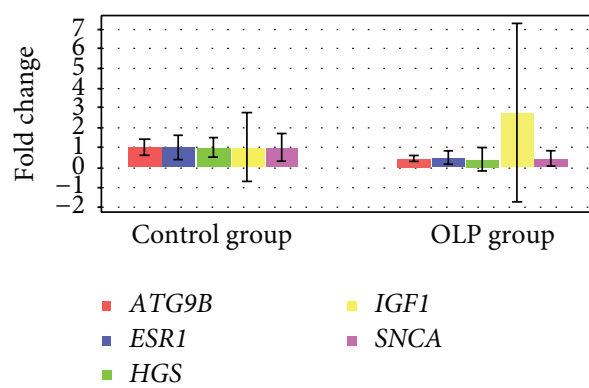

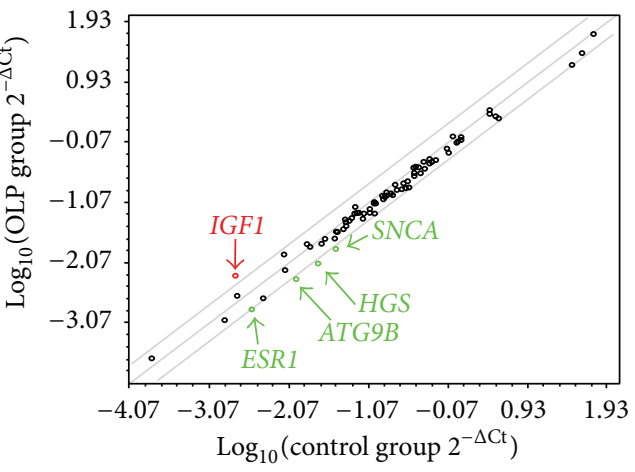

(b)

(d)

FIGURE 1: Different expression of autophagy-associated genes in T cells of OLP. (a) Heat plot displayed different expression of autophagyassociated genes in peripheral blood T cells from OLP patients and controls. (b) Scatter plot showed expression of autophagy-associated genes, with a fold difference of 2. (c) Volcano plot showed the significant difference in expression of autophagy-associated genes on T cells between OLP patients and control individuals. Fold change and fold regulation bigger than 2 were highlighted in red; fold change less than 0.5 and fold regulation less than -2 were highlighted in green. (d) Multianalysis displayed obvious overexpressed IGF1 mRNA and decreased expression of ATG9B, HGS, ESR1, and SNCA in T cells of OLP.

SNCA mRNA expression in T cells showed no significant differences between male $(n=10)$ and female OLP $(n=$ 12) patients or between female OLP patients and female controls (Figures 4(b) and 4(a)). No significant differences in IGF1, ATG9B, HGS, ESR1, and SNCA mRNA expression were detected between male OLP patients and male controls or between male $(n=4)$ and female $(n=6)$ controls (Figures $4(\mathrm{c})$ and $4(\mathrm{~d}))$.
3.5. Differences in the Expression of IGF1, ATG9B, HGS, ESR1, and SNCA in T Cells among OLP Patients of Different Ages. Notably, IGF1 mRNA expression was elevated in middleaged (30-50 years old) OLP patients (OLP $(n=13)$ versus control $(n=4): 1.63 \pm 0.37$ versus $0.7 \pm 0.15$, $p=0.035)$. However, IGF1 mRNA expression was not significantly different between OLP patients aged $<30$ years $(n=4)$ and $>50$ years $(n=5)$ (Figure $5(\mathrm{a}))$. No significant 


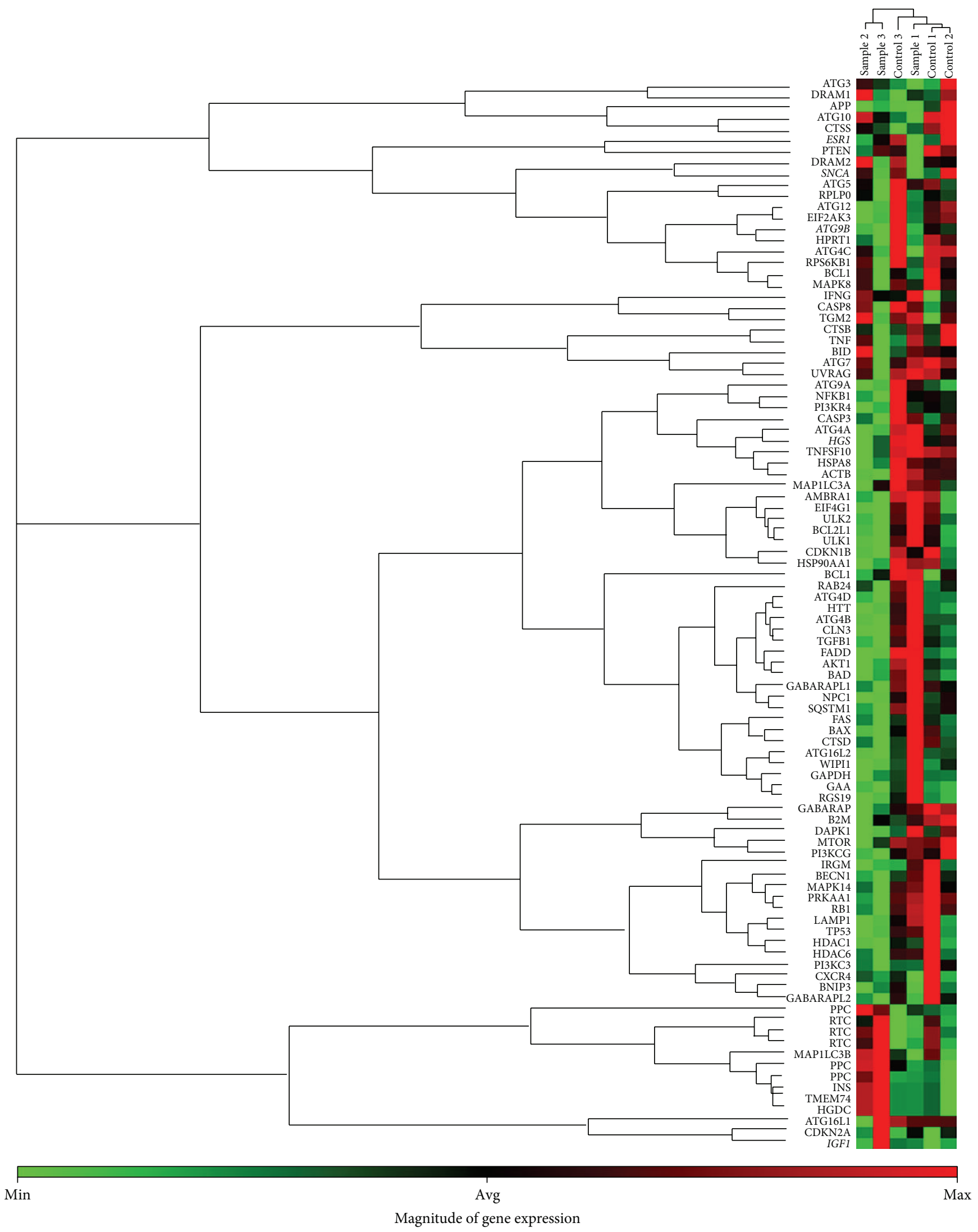

FIGURE 2: Expression patterns of autophagy-associated genes in OLP patients and controls based on autophagy array. Expression clusters were indicated by color bars next to the genes and subjects that were included in different clusters. The magnitude of gene expression was present at the bottom of the figure, which represented the gene mRNA relative expression levels by a range of color. Different autophagy-associated genes expression profiles in peripheral blood T cells were shown in two OLP forms and controls (sample 1: nonerosive OLP, NEOLP: sample 2 and sample 3: erosive OLP, EOLP, and control 1, control 2, and control 3: healthy controls). 


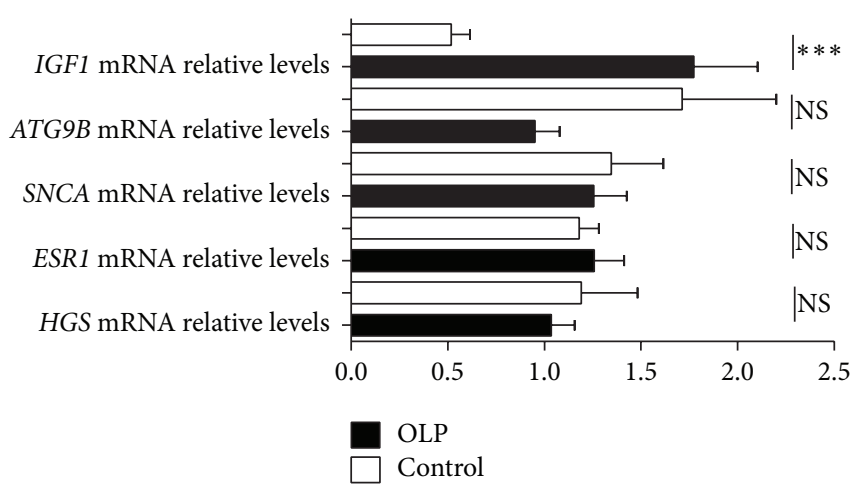

(a)

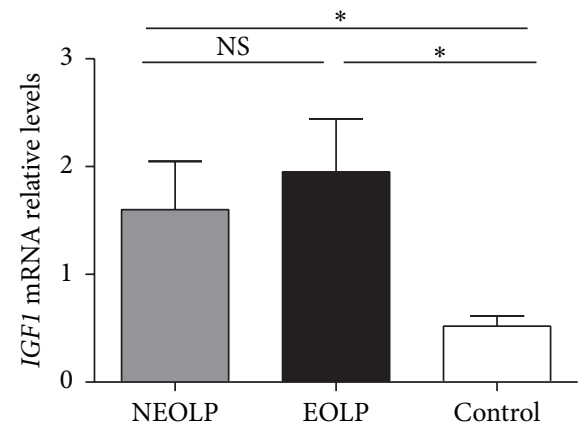

(c)

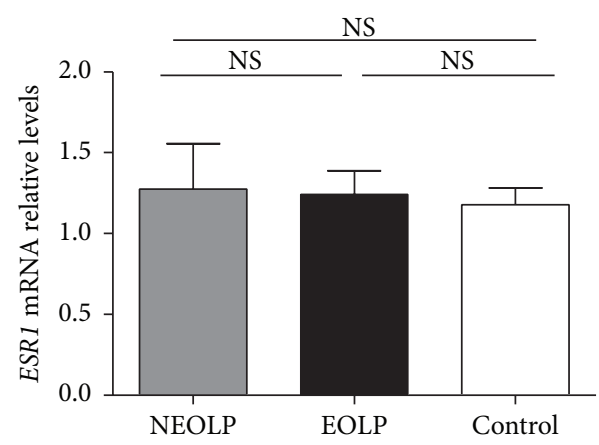

(e)

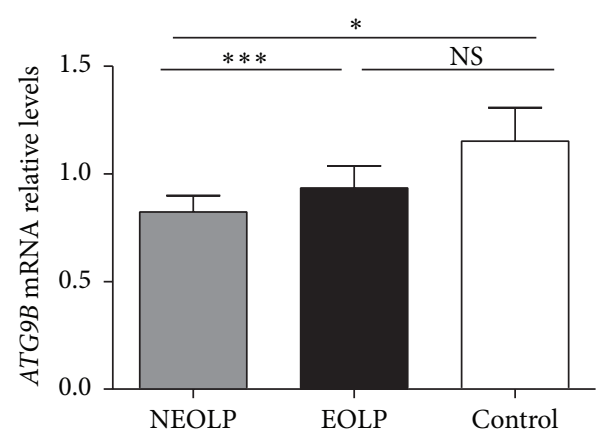

(b)

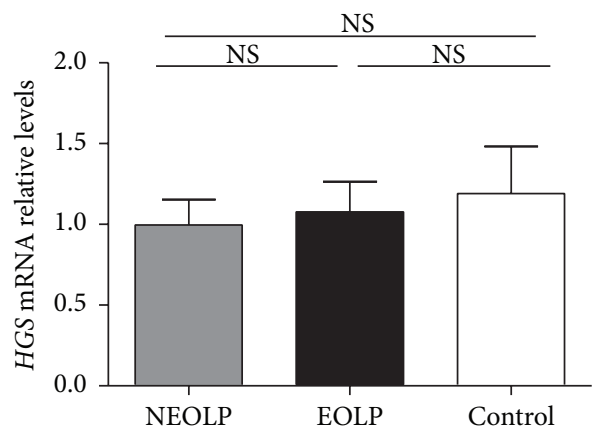

(d)

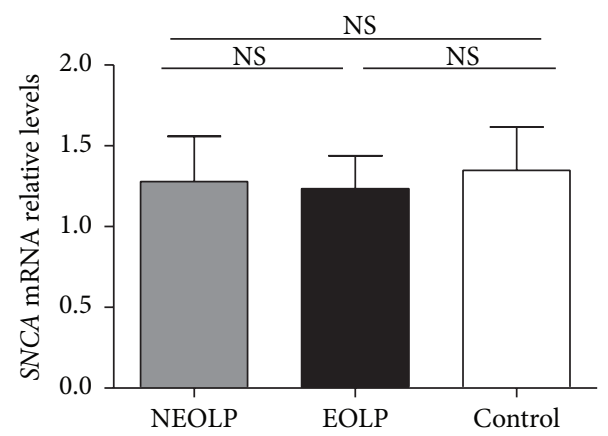

(f)

FIGURE 3: Expression of IGF1, ATG9B, HGS, ESR1, and SNCA of T cells in OLP patients and controls. (a) Expression of IGF1, ATG9B, HGS, ESR1, and SNCA mRNA in T cells of OLP patients $(n=22)$ and controls $(n=10)$. (b-f) The mRNA expression of IGF1, ATG9B, HGS, ESR1, and SNCA in nonerosive OLP patients (NEOLP, $n=11$ ), erosive OLP patients (EOLP, $n=11$ ), and healthy controls $(n=10)$. Data were shown as mean $\pm \operatorname{SEM}\left({ }^{* * *} p \leq 0.001,{ }^{*} p<0.05\right.$, NS: nonsignificantly).

differences were found in ATG9B, HGS, ESR1, and SNCA mRNA expression between OLP patients and controls in all age groups (Figures $5(\mathrm{~b})-5(\mathrm{e})$ ).

\section{Discussion}

Accumulating evidence has shown that autophagy-associated genes may regulate immune signaling in a cell type specific way [15], but the role of autophagy in the T cells of OLP patients is still unknown. The analysis of autophagyassociated genes expression using RT-PCR arrays can provide insights into the autophagic function in the cells of patients, which can help to generate new hypotheses concerning the pathogenesis of complex disorders [23]. The array screening approach greatly shortens the time needed to analyze gene expression in complex biological systems, which may lead to the discovery of potential biomarkers for disease maintenance or offer clues for the development of novel therapies. The results of the autophagy-associated gene expression array used in this study demonstrated an altered expression of autophagy-associated genes in the T cells of OLP patients.

The present study showed that IGF1 expression was upregulated in the peripheral blood T cells of OLP patients. IGF1 signaling could reduce cell death and control the potential exacerbation of the autophagic response under conditions of nutritional stress [24-26]. As an important 


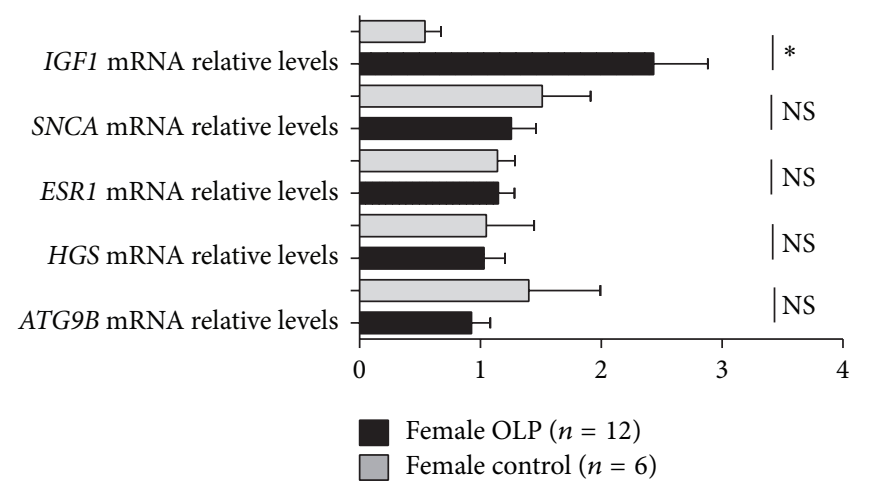

(a)

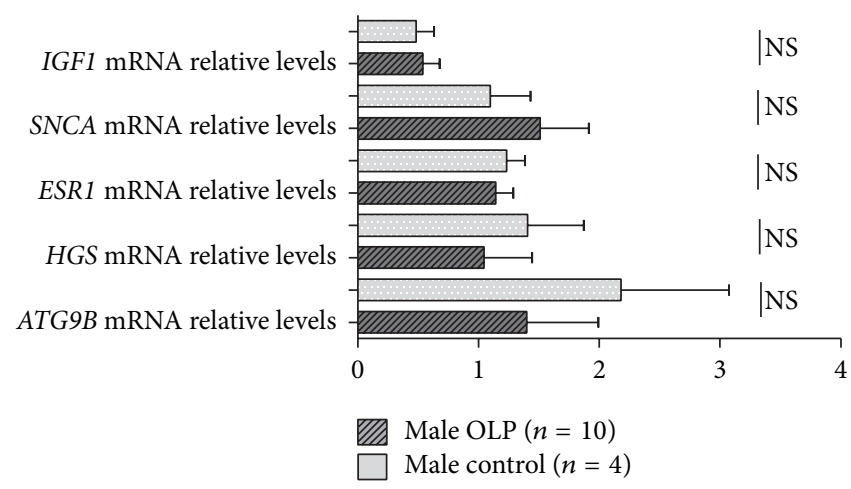

(c)

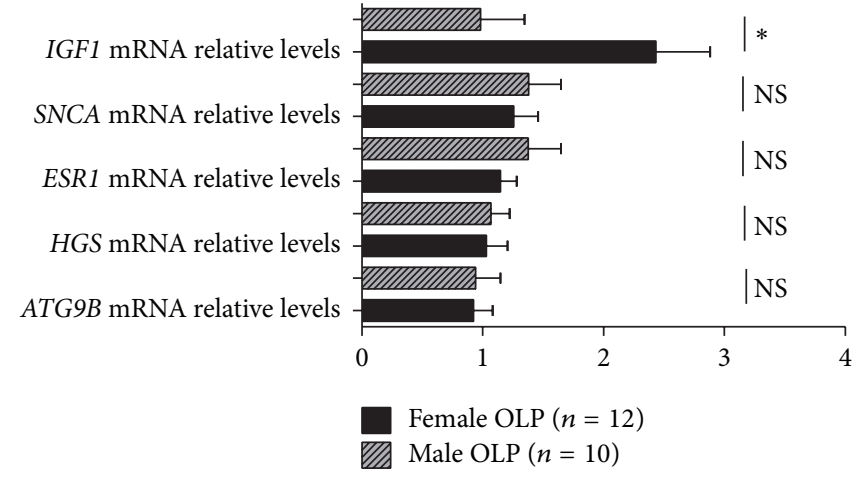

(b)

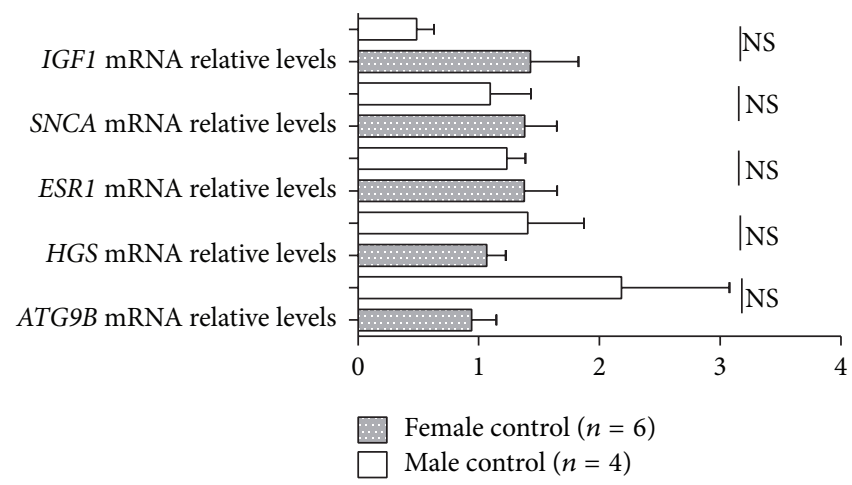

(d)

FIgURE 4: Expression of IGF1, ATG9B, HGS, ESR1, and SNCA mRNA in T cells from different genders of OLP patients and controls. Data were presented as mean $\pm \operatorname{SEM}\left({ }^{*} p<0.05\right.$; NS: nonsignificantly).

regulator of autophagy, IGF1 can modulate the immune functions of peripheral lymphocytes [27]. Previous studies found that a chronic elevation of $I G F 1$ expression exacerbated mouse experimental autoimmune encephalomyelitis (EVE), which might be partly mediated by the expansion of T cells [27]. Moreover, IGF1 and its signaling axis have the potential to enhance myelin-specific $\mathrm{T}$ cell responses and impact the differentiation of $\mathrm{CD}^{+}{ }^{+} \mathrm{T}$ cells into subsets such as Th1 and Th17 cells in EVE $[27,28]$. Increased IGF1 expression might also regulate the activation of $\mathrm{CD}^{+} \mathrm{T}$ cells by inducing p53 gene hypermethylation and could contribute to the polarization of antigen-specific $\mathrm{CD}^{+} \mathrm{T}$ cells in asthma [29]. Our previous studies implicated an imbalance between the Th1/Th2 immune response in OLP and showed that these responses had a predominant Th1 bias $[4,6,7]$. We detected the expression of two Th1/Th2-specific transcription factors, T-bet and GATA-3, in peripheral blood mononuclear cells and found that the expression of T-bet and the ratio of T-bet/GATA-3 mRNA in OLP subjects were significantly higher than those in controls [7]. We also obtained results suggesting that OLP may be characterized by elevations of Th1 chemokines and cytokines, including C-C chemokine receptor 5 (CCR5), interleukin- (IL-) 2, and interferon(IFN-) $\gamma[4,6]$. In addition, Th17 cells and Th17-associated cytokines may be involved in the immune regulation of OLP [8]. Based on these findings, we speculated that the increased expression of IGF1 in T cells from patients with OLP may mediate the immune response in OLP by regulating the T cell proliferation, differentiation, and activation.

Interestingly, the present data indicated a higher IGF1 mRNA expression in the peripheral blood $\mathrm{T}$ cells of female and middle-aged OLP patients, compared with males and other age groups, respectively. Circulating IGFs are important regulators of prenatal and postnatal growth, and their abundances vary with genders and ages [30]. IGF1 was also found to be differentially expressed in condylar cartilage from a rat model of malocclusion among different genders and age groups [31]. Previous studies, including ours, have identified variations in the immunoreactivity of OLP among different genders and age groups [7]. OLP affects women more frequently than men, at a ratio of approximately $1.4: 1$ and occurs predominantly in middle-aged adults [3]. Taken together, we conjectured that IGF1 expression in T cells may contribute to OLP in a gender-dependent and age-associated manner.

ATG9 is the integral multispan transmembrane protein among the core ATG proteins, which are required for autophagosome formation [32, 33]. The functions of ATG9 include the regulation of autophagy and the inhibition of innate immune signaling [12]. T cell autophagy appears to be abnormally regulated in autoimmune diseases such as multiple sclerosis and systemic lupus erythematosus [34, 35]. The array analysis performed in the present study showed that $A T G 9 B$ expression in T cells was lower in OLP patients 


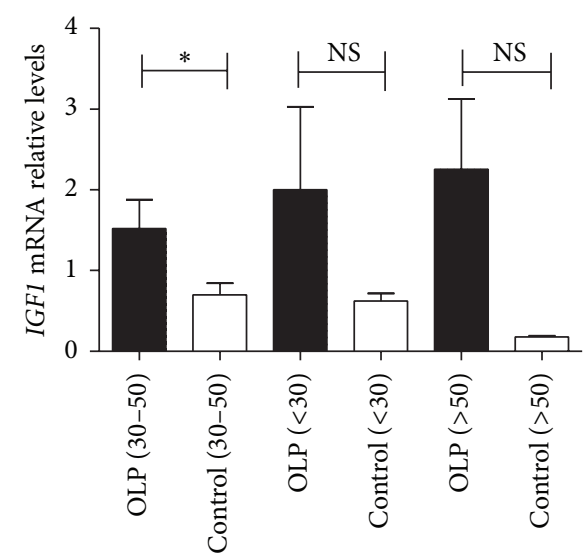

(a)

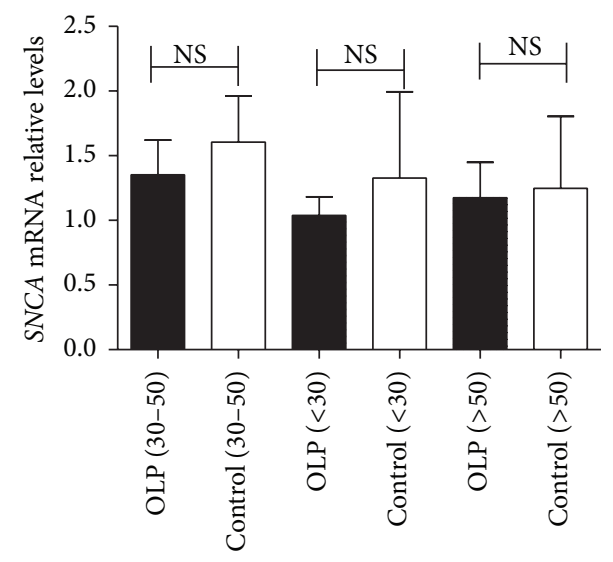

(c)

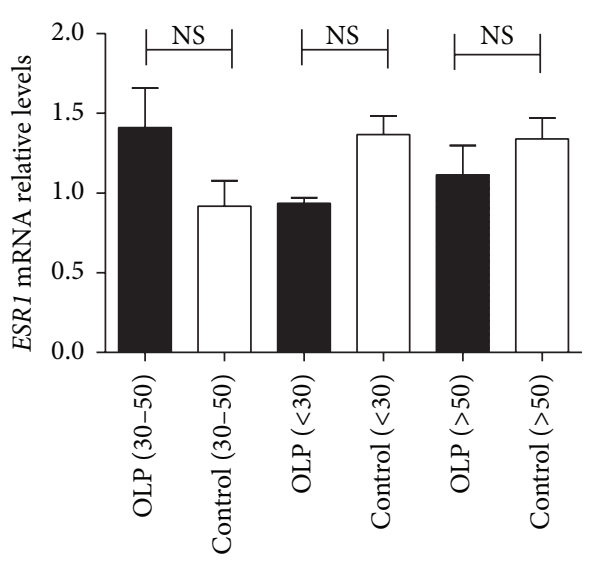

(b)

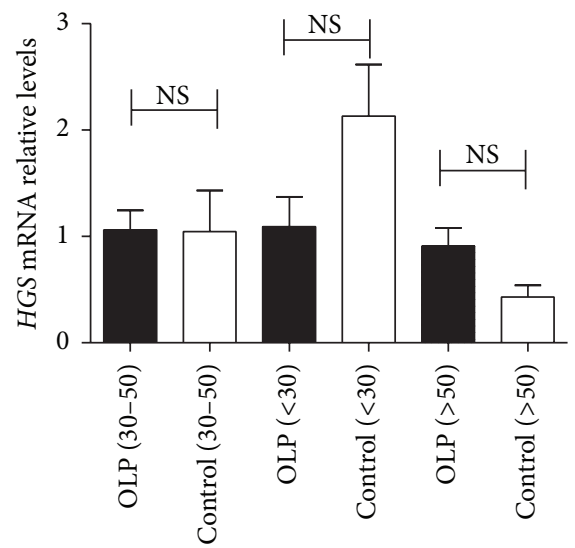

(d)

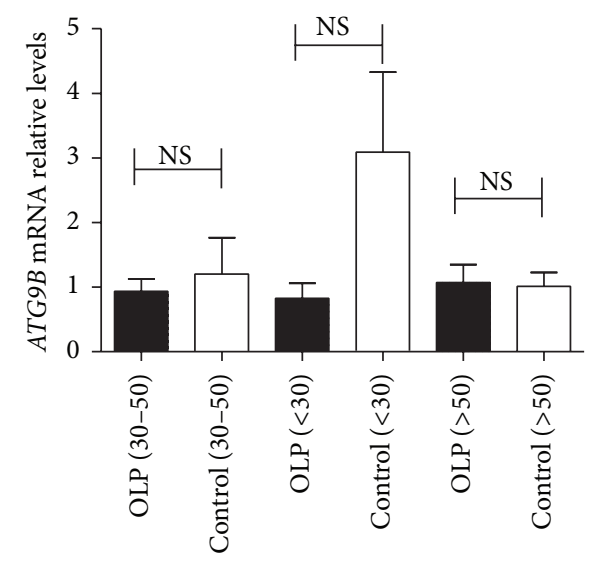

(e)

Figure 5: Expression of IGF1, ATG9B, HGS, ESR1, and SNCA mRNA in T cells from different age groups of OLP patients. The subjects were divided into three age groups, including those aged $<30$ (OLP patients $[n=4]$ versus controls $[n=3]$ ), those aged 30-50 years old (OLP patients $[n=13]$ versus controls $[n=4]$ ), and those aged $>50$ (OLP patients $[n=5]$ versus controls $[n=3]$ ). Data were shown as mean \pm SEM $\left({ }^{*} p<0.05\right.$, NS: nonsignificantly).

than in controls. A reduced expression of $A T G 9 B$, to some extent, might lead to autophagy dysregulation in the T cells of OLP patients. Notably, the mRNA expression of ATG9B in $T$ cells was significantly decreased in patients with nonerosive OLP, when compared with that in controls and patients with erosive OLP. ATG9B, also known as endothelial nitricoxide synthase antisense, is robustly induced by hypoxia [36]. Under hypoxic conditions, oral mucosa epithelial cells mainly undergo apoptosis in the lesion area of OLP [37]. The expression clusters of autophagy-associated genes in 
OLP suggested that differences in T cell autophagic activity may be associated with the different clinical presentation. Besides mediating the survival and homeostasis of cells, autophagy has been implicated both in antigen processing and presentation and in the secretion of proinflammatory cytokines such as type I IFN and tumor necrosis factor(TNF-) $\alpha$ [12]. This latter function of autophagy is especially important in OLP, given that $\mathrm{T}$ cells regulate the apoptosis of basal keratinocytes via the secretion of TNF- $\alpha$, especially in the more advanced stages of OLP [38]. Erosive OLP, which is featured by more evident oral mucosal damage, is generally considered to be a more severe form than nonerosive OLP [39]. Thus, we speculated that the decreased expression of ATG9B in T cells of nonerosive OLP patients might represent compromised autophagy, which could lead to less apoptosis of keratinocytes. In conclusion, ATG9B in T cells of OLP may contribute to the epithelial damage in different clinical forms.

The downregulation of other autophagy-associated genes including HGS, ESR1, and SNCA in peripheral blood T cells of OLP patients was indicated by the array analysis. $H G S$ encodes a protein that regulates endosomal sorting and plays a critical role in the lysosomal transport, recycling, and degradation of ubiquitinated membrane proteins in the multivesicular body, which may be a key machinery for autophagic regulation [40]. ESR1 is essential for sexual development and reproductive function and is involved in the induction of autophagy in response to toxins [41, 42]. Autophagy inhibition can reduce intracellular SNCA aggregation and enhance SNCA secretion, which leads to an exacerbation of the microenvironmental response, including inflammation and cellular damage [43]. However, the confirmatory experiments using quantitative real-time RTPCR showed no significant differences in the expression of HGS, ESR1, and SNCA in the peripheral blood T cells of OLP patients. These results suggested that a larger cohort of patients might yield further insights into autophagyassociated gene expression in OLP.

\section{Conclusion}

In summary, this study is the first to have analyzed the mRNA expression of autophagy-associated genes in peripheral $\mathrm{T}$ cells of OLP patients using autophagy array technology. Our results revealed an upregulation of $I G F 1$ expression on $\mathrm{T}$ cells in a gender-dependent and age-associated manner and a distinct expression pattern of $A T G 9 B$ between different clinical forms, which may suggest the role of autophagy in the immune response of OLP. Further studies concerning the mechanisms of IGF1 and ATG9B in T cells autophagy of OLP are needed and may provide new therapeutic strategies for OLP.

\section{Conflict of Interests}

All authors declare no conflict of interests regarding the publication of this paper.

\section{Acknowledgment}

This work was supported by a grant from the National Natural Science Foundation of China (no. 81371147) to Professor Gang Zhou.

\section{References}

[1] D. Farhi and N. Dupin, "Pathophysiology, etiologic factors, and clinical management of oral lichen planus, part I: facts and controversies," Clinics in Dermatology, vol. 28, no. 1, pp. 100$108,2010$.

[2] J. Zhang and G. Zhou, "Green tea consumption: an alternative approach to managing oral lichen planus," Inflammation Research, vol. 61, no. 6, pp. 535-539, 2012.

[3] M. R. Roopashree, R. V. Gondhalekar, M. C. Shashikanth, J. George, S. H. Thippeswamy, and A. Shukla, "Pathogenesis of oral lichen planus-a review," Journal of Oral Pathology \& Medicine, vol. 39, no. 10, pp. 729-734, 2010.

[4] G. Zhou, J. Zhang, X.-W. Ren, J.-Y. Hu, G.-F. Du, and X.-Y. $\mathrm{Xu}$, "Increased B7-H1 expression on peripheral blood T cells in oral lichen planus correlated with disease severity," Journal of Clinical Immunology, vol. 32, no. 4, pp. 794-801, 2012.

[5] G. Lorenzini, M. Viviano, E. Chisci, G. Chisci, and M. Picciotti, "A comparative immunohistochemical and immunophenotypical study on lymphocytes expression in patients affected by oral lichen planus," Journal of Oral Pathology \& Medicine, vol. 42, no. 8, pp. 642-647, 2013.

[6] J.-Y. Hu, J. Zhang, J.-L. Cui et al., "Increasing CCL5/CCR5 on $\mathrm{CD}^{+} \mathrm{T}$ cells in peripheral blood of oral lichen planus," Cytokine, vol. 62, no. 1, pp. 141-145, 2013.

[7] R. Lu, G. Zhou, G. Du, X. Xu, J. Yang, and J. Hu, "Expression of T-bet and GATA-3 in peripheral blood mononuclear cells of patients with oral lichen planus," Archives of Oral Biology, vol. 56, no. 5, pp. 499-505, 2011.

[8] M.-P. Piccinni, L. Lombardelli, F. Logiodice et al., "Potential pathogenetic role of Th17, Th0, and Th2 cells in erosive and reticular oral lichen planus," Oral Diseases, vol. 20, no. 2, pp. 212-218, 2014.

[9] X.-A. Tao, J. Xia, X.-B. Chen et al., "FOXP3 ${ }^{+}$T regulatory cells in lesions of oral lichen planus correlated with disease activity," Oral Diseases, vol. 16, no. 1, pp. 76-82, 2010.

[10] S. Wang, P. Xia, M. Rehm, and Z. Fan, "Autophagy and cell reprogramming," Cellular and Molecular Life Sciences, vol. 72, no. 9, pp. 1699-1713, 2015.

[11] C. Münz, "Enhancing immunity through autophagy," Annual Review of Immunology, vol. 27, no. 1, pp. 423-449, 2009.

[12] B. Levine, N. Mizushima, and H. W. Virgin, "Autophagy in immunity and inflammation," Nature, vol. 469, no. 7330, pp. 323-335, 2011.

[13] C. Li, E. Capan, Y. Zhao et al., "Autophagy is induced in $\mathrm{CD} 4^{+} \mathrm{T}$ cells and important for the growth factor-withdrawal cell death," The Journal of Immunology, vol. 177, no. 8, pp. 5163-5168, 2006.

[14] R. Watanabe, H. Fujii, T. Shirai, S. Saito, T. Ishii, and H. Harigae, "Autophagy plays a protective role as an anti-oxidant system in human T cells and represents a novel strategy for induction of T-cell apoptosis," European Journal of Immunology, vol. 44, no. 8, pp. 2508-2520, 2014.

[15] H. W. Virgin and B. Levine, "Autophagy genes in immunity," Nature Immunology, vol. 10, no. 5, pp. 461-470, 2009. 
[16] G. L. C. Paulus and R. J. Xavier, "Autophagy and checkpoints for intracellular pathogen defense," Current Opinion in Gastroenterology, vol. 31, no. 1, pp. 14-23, 2015.

[17] H. Cheong, "Integrating autophagy and metabolism in cancer," Archives of Pharmacal Research, vol. 38, no. 3, pp. 358-371, 2015.

[18] B. Levine and G. Kroemer, "Autophagy in the pathogenesis of disease," Cell, vol. 132, no. 1, pp. 27-42, 2008.

[19] J. Joven, M. Guirro, R. Mariné-Casadó, E. Rodríguez-Gallego, and J. A. Menéndez, "Autophagy is an inflammation-related defensive mechanism against disease," Advances in Experimental Medicine and Biology, vol. 824, pp. 43-59, 2014.

[20] J. C. Barrett, S. Hansoul, D. L. Nicolae et al., "Genome-wide association defines more than 30 distinct susceptibility loci for Crohn's disease," Nature Genetics, vol. 40, no. 8, pp. 955-962, 2008.

[21] M. Alirezaei, H. S. Fox, C. T. Flynn et al., "Elevated ATG5 expression in autoimmune demyelination and multiple sclerosis," Autophagy, vol. 5, no. 2, pp. 152-158, 2009.

[22] X.-J. Zhou and H. Zhang, "Autophagy in immunity: implications in etiology of autoimmune/autoinflammatory diseases," Autophagy, vol. 8, no. 9, pp. 1286-1299, 2012.

[23] Q. Jiang, Y. Wang, T. Li et al., "Heat shock protein 90-mediated inactivation of nuclear factor- $\kappa \mathrm{B}$ switches autophagy to apoptosis through becnl transcriptional inhibition in selenite-induced NB4 cells," Molecular Biology of the Cell, vol. 22, no. 8, pp. 11671180, 2011.

[24] R. Troncoso, J. M. Vicencio, V. Parra et al., "Energypreserving effects of IGF-1 antagonize starvation-induced cardiac autophagy," Cardiovascular Research, vol. 93, no. 2, pp. 320329, 2012.

[25] R. Troncoso, J. Díaz-Elizondo, S. P. Espinoza et al., "Regulation of cardiac autophagy by insulin-like growth factor 1," IUBMB Life, vol. 65, no. 7, pp. 593-601, 2013.

[26] Y. Gu, C. Wang, and A. Cohen, "Effect of IGF-1 on the balance between autophagy of dysfunctional mitochondria and apoptosis," FEBS Letters, vol. 577, no. 3, pp. 357-360, 2004.

[27] S. Genoud, I. Maricic, V. Kumar, and F. H. Gage, "Targeted expression of IGF-1 in the central nervous system fails to protect mice from experimental autoimmune encephalomyelitis," Journal of Neuroimmunology, vol. 168, no. 1-2, pp. 40-45, 2005.

[28] M. F. Cusick, J. E. Libbey, N. S. Trede, and R. S. Fujinami, "Targeting insulin-like growth factor 1 leads to amelioration of inflammatory demyelinating disease," PLoS ONE, vol. 9, no. 4, Article ID e94486, 2014.

[29] J.-Y. Zou, S.-H. Huang, Y. Li, H.-G. Chen, J. Rong, and S. Ye, "Airway epithelial cell-derived insulin-like growth factor-1 triggers skewed $\mathrm{CD}^{+} \mathrm{T}$ cell polarization," Cell Biology International, vol. 38, no. 10, pp. 1148-1154, 2014.

[30] K. L. Gatford, G. K. Heinemann, S. D. Thompson et al., "Circulating IGF1 and IGF2 and SNP genotypes in men and pregnant and non-pregnant women," Endocrine Connections, vol. 3, no. 3, pp. 138-149, 2014.

[31] S. Yu, L. Sun, L. Liu, K. Jiao, and M. Wang, "Differential expression of IGF1, IGFR1 and IGFBP3 in mandibular condylar cartilage between male and female rats applied with malocclusion," Journal of Oral Rehabilitation, vol. 39, no. 10, pp. 727-736, 2012.

[32] N. Mizushima, T. Yoshimori, and Y. Ohsumi, "The role of atg proteins in autophagosome formation," Annual Review of Cell and Developmental Biology, vol. 27, pp. 107-132, 2011.
[33] H. Nakatogawa, K. Suzuki, Y. Kamada, and Y. Ohsumi, "Dynamics and diversity in autophagy mechanisms: lessons from yeast," Nature Reviews Molecular Cell Biology, vol. 10, no. 7, pp. 458-467, 2009.

[34] M. Pierdominici, M. Vomero, C. Barbati et al., "Role of autophagy in immunity and autoimmunity, with a special focus on systemic lupus erythematosus," The FASEB Journal, vol. 26, no. 4, pp. 1400-1412, 2012.

[35] A. Bhattacharya and N. T. Eissa, "Autophagy and autoimmunity crosstalks," Frontiers in Immunology, vol. 4, article 88, 2013.

[36] J. E. Fish, C. C. Matouk, E. Yeboah et al., "Hypoxiainducible expression of a natural cis-antisense transcript inhibits endothelial nitric-oxide synthase," The Journal of Biological Chemistry, vol. 282, no. 21, pp. 15652-15666, 2007.

[37] M. Ding, J. Y. Xu, and Y. Fan, "Altered expression of mRNA for HIF- $1 \alpha$ and its target genes RTP801and VEGF in patients with oral lichen planus," Oral Diseases, vol. 16, no. 3, pp. 299-304, 2010.

[38] M. R. Payeras, K. Cherubini, M. A. Figueiredo, and F. G. Salum, "Oral lichen planus: focus on etiopathogenesis," Archives of Oral Biology, vol. 58, no. 9, pp. 1057-1069, 2013.

[39] X. Tao, Y. Huang, R. Li et al., "Assessment of local angiogenesis and vascular endothelial growth factor in the patients with atrophic-erosive and reticular oral lichen planus," Oral Surgery, Oral Medicine, Oral Pathology, Oral Radiology and Endodontology, vol. 103, no. 5, pp. 661-669, 2007.

[40] S. Miura and Y. Mishina, "Hepatocyte growth factor-regulated tyrosine kinase substrate (Hgs) is involved in BMP signaling through phosphorylation of smads and TAK1 in early mouse embryo," Developmental Dynamics, vol. 240, no. 11, pp. 24742481, 2011.

[41] Y. Chen and R. G. Xia, "Screening and functional microarray analysis of differentially expressed genes related to osteoporosis," Genetics and Molecular Research, vol. 13, no. 2, pp. 32283236, 2014.

[42] K. Williams, K. Gokulan, D. Shelman, T. Akiyama, A. Khan, and S. Khare, "Cytotoxic mechanism of cytolethal distending toxin in nontyphoidal salmonella serovar (salmonella javiana) during macrophage infection," DNA and Cell Biology, vol. 34, no. 2, pp. 113-124, 2015.

[43] A.-M. Poehler, W. Xiang, P. Spitzer et al., "Autophagy modulates SNCA $/ \alpha$-synuclein release, thereby generating a hostile microenvironment," Autophagy, vol. 10, no. 12, pp. 2171-2192, 2014. 


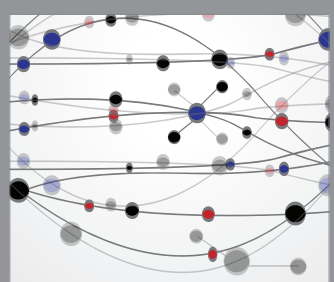

The Scientific World Journal
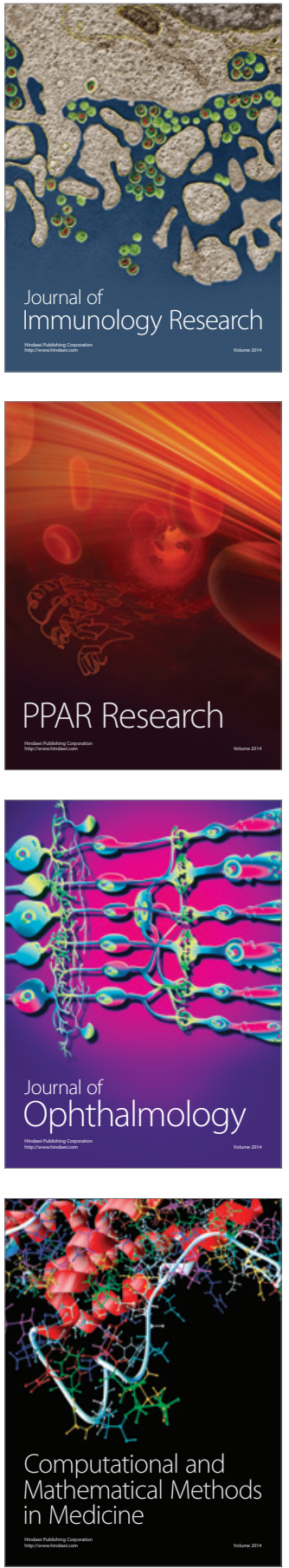

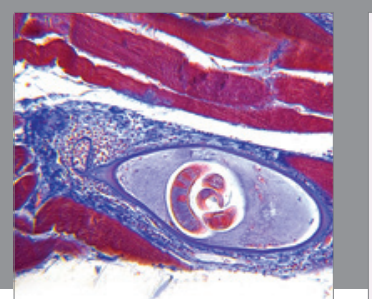

Gastroenterology Research and Practice

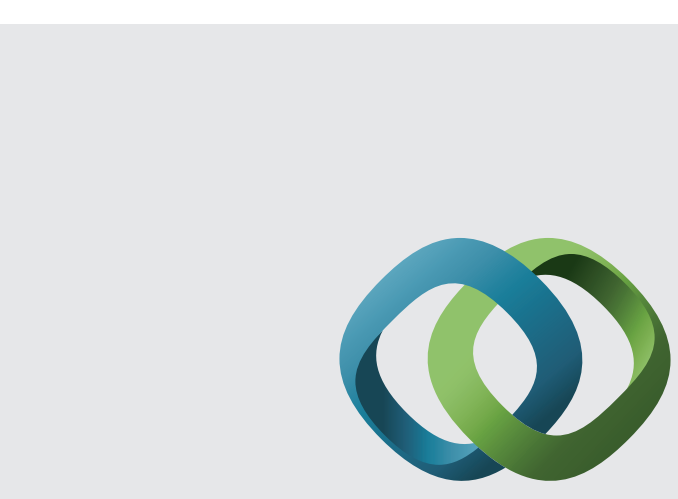

\section{Hindawi}

Submit your manuscripts at

http://www.hindawi.com
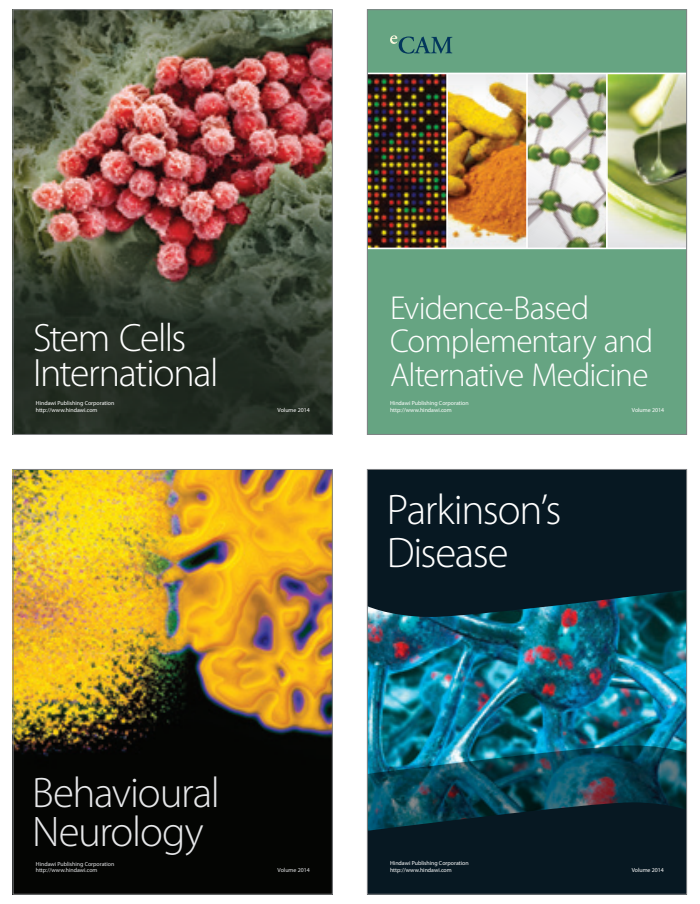
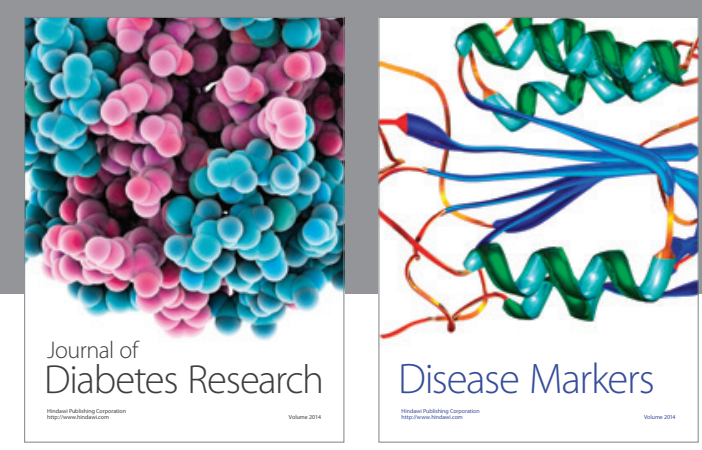

Disease Markers
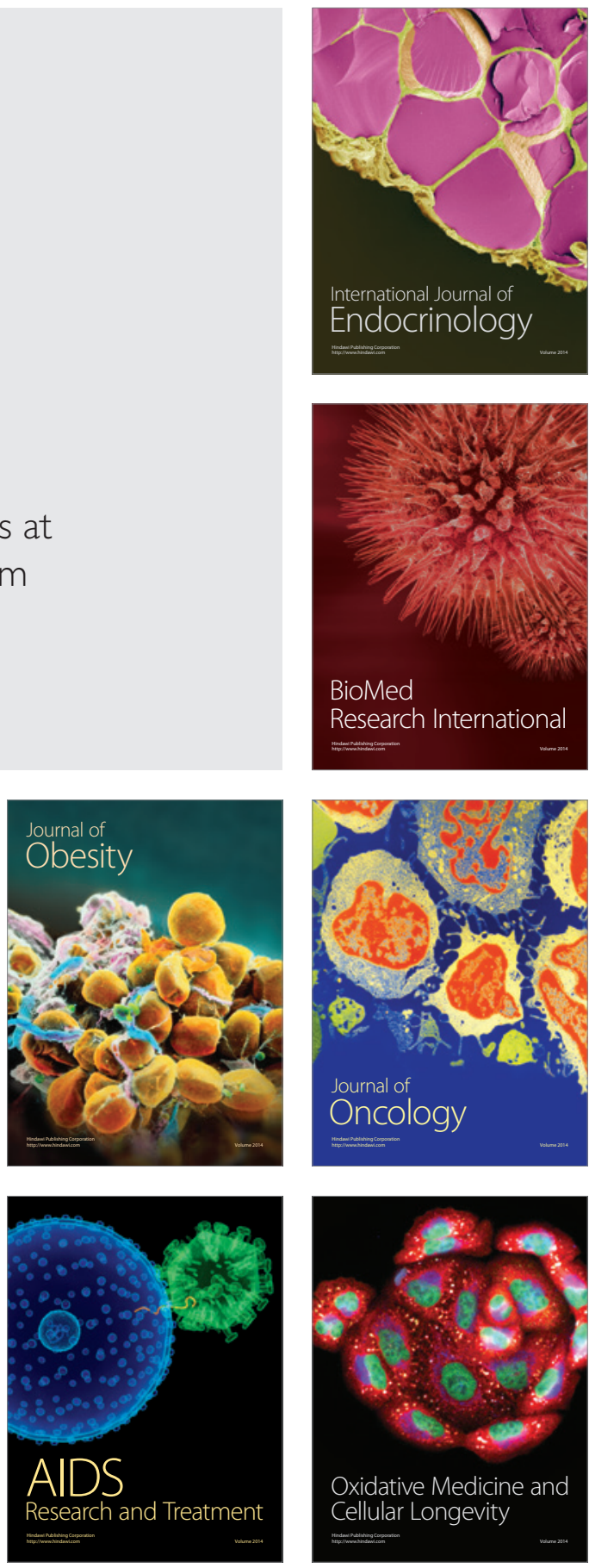